\title{
Constraints on "Fixed Point" QCD from the CCFR Data on Deep Inelastic Neutrino-Nucleon Scattering
}

\author{
Aleksander V. Sidorov \\ Bogoliubov Theoretical Laboratory \\ Joint Institute for Nuclear Research \\ 141980 Dubna, Russia \\ E-mail: sidorov@thsun1.jinr.dubna.su \\ Dimiter B. Stamenov f \\ International Centre for Theoretical Physics, Trieste, Italy
}

\begin{abstract}
The results of LO Fixed point QCD (FP-QCD) analysis of the CCFR data for the nucleon structure function $x F_{3}\left(x, Q^{2}\right)$ are presented. The predictions of FP-QCD, in which $\alpha_{s}\left(Q^{2}\right)$ tends to a nonzero coupling constant $\alpha_{0}$ as $Q^{2} \rightarrow \infty$, are in good agreement with the data. Constraints for the possible values of the $\beta$ function parameter $b$ regulating how fast $\alpha_{s}\left(Q^{2}\right)$ tends to its asymptotic value $\alpha_{0}$ are found from the data. The corresponding values of $\alpha_{0}$ are also determined. Having in mind the recent QCD fits to the same data we conclude that in spite of the high precision and the large $\left(x, Q^{2}\right)$ kinematic range of the CCFR data they cannot discriminate between QCD and FP-QCD predictions for $x F_{3}\left(x, Q^{2}\right)$.
\end{abstract}

* Institute for Nuclear Research and Nuclear Energy, Bulgarian Academy of Sciences Boul. Tsarigradsko chaussee 72, Sofia 1784, Bulgaria. E-mail:stamenov@bgearn.bitnet 


\section{Introduction.}

The success of perturbative Quantum Chromodynamics (QCD) in the description of the high energy physics of strong interactions is considerable. The QCD predictions are in good quantitative agreement with a great number of data on lepton-hadron and hadron-hadron processes in a large kinematic region (e.g. see reviews [1] and the references therein). Despite of this success of QCD, we consider that it is useful and reasonable to put the question: Do the present data fully exclude the so-called fixed point (FP) theory models [2] ?

We remind that these models are not asymptotically free. The effective coupling constant $\alpha_{s}\left(Q^{2}\right)$ approaches a constant value $\alpha_{0} \neq 0$ as $Q^{2} \rightarrow \infty$ (the so-called fixed point at which the Callan- Symanzik $\beta$-function $\left.\beta\left(\alpha_{0}\right)=0\right)$. Using the assumption that $\alpha_{0}$ is small one can make predictions for the physical quantities in the high energy region, as like in QCD, and confront them to the experimental data. Such a test of FP

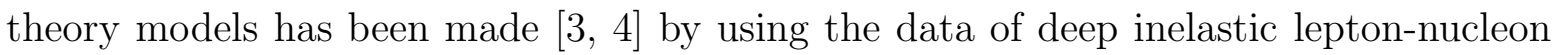
experiments started by the SLAC-MIT group [5] at the end of the sixties and performed in the seventies [6]. It was shown that

i) the predictions of the FP theory models with scalar and non-colored (Abelian) vector gluons do not agree with the data

ii) the data cannot distinguish between different forms of scaling violation predicted by QCD and the so-called Fixed point QCD (FP-QCD), a theory with colored vector

gluons, in which the effective coupling constant $\alpha_{s}\left(Q^{2}\right)$ does not vanish when $Q^{2}$ tends to infinity.

We think there are two reasons to discuss again the predictions of FP-QCD. First of all, there is evidence from the non-perturbative lattice calculations [7] that the $\beta$ function in QCD vanishes at a nonzero coupling $\alpha_{0}$ that is small. (Note that the structure of the $\beta$-function can be studied only by non-perturbative methods.) Secondly, in the last years the accuracy and the kinematic region of deep inelastic scattering data became large enough, which makes us hope that discrimination between QCD and FPQCD could be performed.

Recently we have analyzed the CCFR deep inelastic neutrino-nucleon scattering data [8] in the framework of the Fixed point QCD. It was demonstrated [9] that the data for the nucleon structure function $x F_{3}\left(x, Q^{2}\right)$ are in good agreement with the LO predictions of this theory model using the assumption that the fixed point coupling 
$\alpha_{0}$ is small. In contrast to the results of the fits to the previous generations of deep inelastic lepton-nucleon experiments, the value of this constant was determined with a good accuracy:

$$
\alpha_{0}=0.198 \pm 0.009
$$

However, this value of $\alpha_{0}$ is not consistent with $\alpha_{s}\left(M_{z}^{2}\right)$ measurements at LEP. For instance, from the scaling violation in the fragmentation functions in $e^{+} e^{-}$annihilation $\alpha_{s}\left(M_{z}^{2}\right)$ has been determined [10] as:

$$
\alpha_{s}\left(M_{z}^{2}\right)=0.118 \pm 0.005
$$

This discrepancy follows from the fact that in our analysis pure asymptotic formula for the structure function $x F_{3}$ has been used, i.e. the effective coupling constant

$\alpha_{s}\left(Q^{2}\right)$ has been approximated with its asymptotic value $\alpha_{0}$. Results (1) and (2) have shown that in the $Q^{2}$ range studied up to now $\alpha_{0}$ is not yet reached and therefore, to determine $\alpha_{0}$ properly from the data the preasymptotic behaviour of $\alpha_{s}\left(Q^{2}\right)$ has to be taken into account.

In this paper we present a leading order Fixed point QCD analysis of the CCFR data [8], in which the next corrections to the pure asymptotic expression for $x F_{3}\left(x, Q^{2}\right)$ are taken into account. We remind that the structure function $x F_{3}$ is a pure non-singlet and the results of the analysis are independent of the assumption on the shape of gluons. As in a previous analysis the method [11] of reconstruction of the structure functions from their Mellin moments is used. This method is based on the Jacobi - polynomial expansion [12] of the structure functions. In [13] this method has been already applied to the QCD analysis of the CCFR data.

\section{Method and Results of Analysis.}

Let us start with the basic formulas needed for our analysis.

The Mellin moments of the structure function $x F_{3}\left(x, Q^{2}\right)$ are defined as:

$$
M_{n}^{N S}\left(Q^{2}\right)=\int_{0}^{1} d x x^{n-2} x F_{3}\left(x, Q^{2}\right)
$$

where $n=2,3,4, \ldots$.

In the case of FP-QCD the effective coupling constant $\alpha_{s}\left(Q^{2}\right)$ at large $Q^{2}$ takes the form:

$$
\alpha_{s}\left(Q^{2}\right)=\alpha_{0}+f\left(Q^{2}\right),
$$

where $f\left(Q^{2}\right) \rightarrow 0$ when $Q^{2} \rightarrow \infty$. 
Let us assume that $\alpha_{0}$ is a first order ultraviolet fixed point for the $\beta$-function, i.e.

$$
\beta(\alpha)=-b\left(\alpha-\alpha_{0}\right), \quad b>0,
$$

Then

$$
\alpha_{s}\left(Q^{2}\right)=\alpha_{0}+\left[\alpha_{s}\left(Q_{0}^{2}\right)-\alpha_{0}\right]\left(\frac{Q_{0}^{2}}{Q^{2}}\right)^{b} .
$$

and instead of Eq.(5) in [9] we obtain now for the moments of $x F_{3}$ the following LO expression:

$$
M_{n}^{N S}\left(Q^{2}\right)=M_{n}^{N S}\left(Q_{0}^{2}\right)\left[\frac{Q_{0}^{2}}{Q^{2}}\right]^{\frac{1}{2} d_{n}^{N S}} F_{n}\left(Q^{2}\right)
$$

where

$$
F_{n}\left(Q^{2}\right)=\exp \left\{\frac{\left(\alpha_{s}\left(Q_{0}^{2}\right)-\alpha_{0}\right)}{2 b \alpha_{0}} d_{n}^{N S}\left[\left(\frac{Q_{0}^{2}}{Q^{2}}\right)^{b}-1\right]\right\} .
$$

In (7) and (8)

$$
d_{n}^{N S}=\frac{\alpha_{0}}{4 \pi} \gamma_{n}^{(0) N S}
$$

and

$$
\gamma_{n}^{(0) N S}=\frac{8}{3}\left[1-\frac{2}{n(n+1)}+4 \sum_{j=2}^{n} \frac{1}{j}\right] .
$$

The $n$ dependence of $\gamma_{n}^{(0) N S}$ is exactly the same as in QCD. However, the $Q^{2}$ behaviour of the moments is different. In contrast to QCD, the Bjorken scaling for the moments of the structure functions is broken by powers in $Q^{2}$. In (8) and (9) $\alpha_{0}$ and $b$ are parameters, to be determined from the data.

Having in hand the moments (7) and following the method [11, 12], we can write the structure function $x F_{3}$ in the form:

$$
x F_{3}^{N_{\max }}\left(x, Q^{2}\right)=x^{\alpha}(1-x)^{\beta} \sum_{n=0}^{N_{\max }} \Theta_{n}^{\alpha, \beta}(x) \sum_{j=0}^{n} c_{j}^{(n)}(\alpha, \beta) M_{j+2}^{N S}\left(Q^{2}\right),
$$

where $\Theta_{n}^{\alpha \beta}(x)$ is a set of Jacobi polynomials and $c_{j}^{n}(\alpha, \beta)$ are coefficients of the series of $\Theta_{n}^{\alpha, \beta}(x)$ in powers in $\mathrm{x}$ :

$$
\Theta_{n}^{\alpha, \beta}(x)=\sum_{j=0}^{n} c_{j}^{(n)}(\alpha, \beta) x^{j} .
$$

$N_{\max }, \alpha$ and $\beta$ have to be chosen so as to achieve the fastest convergence of the series in the R.H.S. of Eq.(11) and to reconstruct $x F_{3}$ with the accuracy required. Following the results of [11 we use $\alpha=0.12, \beta=2.0$ and $N_{\max }=12$. These numbers guarantee accuracy better than $10^{-3}$. 
Finally we have to parametrize the structure function $x F_{3}$ at some fixed value of $Q^{2}=Q_{0}^{2}$. We choose $x F_{3}\left(x, Q^{2}\right)$ in the form:

$$
x F_{3}\left(x, Q_{0}^{2}\right)=A x^{B}(1-x)^{C}
$$

The parameters A, B and C in Eq. (13) and the FP-QCD parameters $\alpha_{0}$ and $b$ are free parameters which are determined by the fit to the data.

In our analysis the target mass corrections [14 are taken into account. To avoid the influence of higher-twist effects we have used only the experimental points in the plane $\left(x, Q^{2}\right)$ with $10<Q^{2} \leq 501(\mathrm{GeV} / \mathrm{c})^{2}$. This cut corresponds to the following $x$ range: $0.015 \leq x \leq 0.65$.

The results of the fit are presented in Table 1. In all fits only statistical errors are taken into account.

\begin{tabular}{|c|c|c|c|c|c|c|}
\hline$b$ & $\chi_{d . f .}^{2}$ & $\alpha_{0}$ & $\mathrm{~A}$ & $\mathrm{~B}$ & $\mathrm{C}$ & $\alpha_{s}\left(M_{z}^{2}\right)$ \\
\hline 0.15 & $82.7 / 61$ & $.057 \pm .026$ & $6.96 \pm .20$ & $.799 \pm .013$ & $3.44 \pm .03$ & $.121 \pm .034$ \\
0.20 & $82.3 / 61$ & $.097 \pm .021$ & $6.95 \pm . .20$ & $.799 \pm .013$ & $3.44 \pm .03$ & $.132 \pm .025$ \\
0.25 & $82.0 / 61$ & $.122 \pm .018$ & $6.94 \pm .19$ & $.798 \pm .013$ & $3.45 \pm .03$ & $.142 \pm .020$ \\
\hline
\end{tabular}

Table 1. The results of the LO FP-QCD fit to the CCFR $x F_{3}$ data. $\chi_{d . f .}^{2}$ is the $\chi^{2}$-parameter normalized to the degree of freedom $d . f$..

Summarizing the results in the Table one can conclude:

1. The values of $\chi_{d . f}^{2}$. are slightly smaller than those obtained in the LO QCD analysis [13] of the CCFR data and indicate a good description of the data.

2. The values of $b$, for which the asymptotic coupling $\alpha_{0}$ is smaller than $\alpha_{s}\left(M_{z}^{2}\right)$, are found to range in the following interval:

$$
0<b<0.25
$$

For the values of $b$ smaller than $0.15 \alpha_{0}$ can not be determined from CCFR data. The errors in $\alpha_{0}$ exceed the mean values of this parameter. For the values of $b \geq 0.25$ the mean value of $\alpha_{0}$ is equal or bigger than $\alpha_{s}\left(M_{z}^{2}\right)$.

3. The accuracy of determination of $\alpha_{0}$ is not good enough. The accuracy increases with increasing $b$.

4. $\alpha_{0}=0.057$ corresponding to $b=0.15$ is preferred to the other values of $\alpha_{0}$ determined from the data. 
5. The values of $\alpha_{s}\left(M_{z}^{2}\right)$ corresponding to the values of $b$ from the range (14) are in agreement within one standard deviation with $\alpha_{s}\left(M_{z}^{2}\right)$ determined from the LEP experiments.

6. The values of the parameters $\mathrm{A}, \mathrm{B}$ and $\mathrm{C}$ are in agreement with the results of [13]. They are found to be independent of $b$ and $\alpha_{0}$. We have found also that multiplying the R.H.S. of $(13)$ by term $(1+\gamma x)$ one can not improve the fit.

\section{Summary.}

The CCFR deep inelastic neutrino-nucleon scattering data have been analyzed in the framework of the Fixed point QCD. It has been demonstrated that the data for the

nucleon structure function $x F_{3}\left(x, Q^{2}\right)$ are in good agreement with the LO predictions of this quantum field theory model using the assumption that $\alpha_{0}$ is a first order ultraviolet fixed point of the $\beta$ function and $\alpha_{0}$ is small. Some constraints on the behaviour of the $\beta$ function near $\alpha_{0}$ have been found from the data. The value $\alpha_{0}=0.057$ corresponding to the $\beta$ function parameter $b=0.15$ we have obtained is preferred to the other ones determined from the data.

In conclusion, we find that the CCFR data, the most precise data on deep inelastic scattering at present, do not eliminate the FP-QCD and therefore other tests have to be made in order to distinguish between QCD and FP-QCD.

\section{Acknowledgement}

We are grateful to Profs. A.L. Kataev, D.I. Kazakov, N.N. Nikolaev, E.A. Paschos, E. Seiler, D.V. Shirkov and N.G. Stefanis for useful discussions and remarks. One of us (D.S) would like to thank also the International Atomic Energy Agency and UNESCO for the hospitality at the International Centre for Theoretical Physics in Trieste where this work was completed.

This research was partly supported by INTAS (International Association for the Promotion of Cooperation with Scientists from the Independent States of the Former Soviet Union) under Contract nb 93-1180, by the Russian Fond for Fundamental Research Grant N 94-02-03463-a and by the Bulgarian Science Foundation under Contract F 16. 


\section{References}

[1] G. Altarelli, in Proc. of the "QCD-20 Years Later" Conf. 9-13 June 1992, Aachen; World Scientific 1993, v. 1., p. 172; Ed. by P. M. Zerwas and H. A. Kastrup.

S. Bethke. Proc. QCD-94 Conference, Montpelier, France, July 1994.

[2] A. M. Polyakov, ZHETF 59 (1970) 542. G. Mack, Nucl. Phys. B35 (1971) 592; A. V. Efremov and I.F. Ginzburg, Phys. Lett. B36 (1972) 371; D. Bailin and A. Love, Nucl. Phys. B75 (1974) 159.

[3] M. Glück and E.Reya, Phys. Rev. D.16 (1977) 3242; Nucl. Phys. B156 (1979) 456.

[4] S. I. Bilenkaya and D. B. Stamenov, Sov. J. of Nucl. Phys. 31 (1980) 122.

[5] D.H.Coward et al., Phys. Rev. Lett. 20 (1968) 292; E.D. Bloom et al., Phys. Rev. Lett. 23 (1969) 930; H. Breidenbach et al., Phys. Rev. Lett. 23 (1969) 935.

[6] R.G. Roberts and M.R. Whalley, J. Phys. G.; Nucl. Part. Phys. 17 (1991) D1D151.

[7] A. Patrascioiu and E. Seiler, Expected Deviations from Perturbative QCD at 1 TeV or Less, preprint MPI-Ph/92-18; Scaling, Asymptotic Scaling and Improved Perturbation Theory, preprint MPI-Ph/93-34; J. Finberg, U. Heller and F. Karsh, Nucl. Phys. B392 (1993) 493.

[8] CCFR Collab., S. R. Mishra et al., Nevis Preprint N 1459 (1992); CCFR Collab., W. C. Leung et al., Phys. Lett. B317 (1993) 655; CCFR Collab., P. Z. Quintas et al., Phys. Rev. Lett. 71 (1993) 1307.

[9] A.V. Sidorov and D. B. Stamenov, Preprint JINR E2-95-107, Dubna, 1995.

[10] DELPHI Collab., P. Abrea et al., Phys. Lett. B311 (1993) 408.

[11] V. G. Krivokhizhin et al., Z. Phys. C36 (1987) 51;

V. G. Krivokhizhin et al., Z. Phys. C48 (1990) 347.

[12] G. Parisi and N. Sourlas, Nucl. Phys. B151 (1979) 421;

I. S. Barker, C. B. Langensiepen and G. Shaw, Nucl. Phys. B186 (1981) 61.

[13] A. L. Kataev and A.V. Sidorov, Phys. Lett. B331 (1994) 179.

[14] O. Nachtmann, Nucl. Phys. B63 (1973) 237;

S. Wandzura, Nucl. Phys. B122 (1977) 412. 